\title{
The yeast two-component SLN1 pathway and the scaffolding activity of Pbs 2 modulate the response to endoplasmic reticulum stress induced by tunicamycin.
}

\section{Mariana Hernández-Elvira}

Universidad Nacional Autonoma de Mexico Instituto de Fisiologia Celular

\section{Griselda Salas-Delgado}

Universidad Nacional Autonoma de Mexico Instituto de Fisiologia Celular

\section{Laura Kawasaki}

Universidad Nacional Autonoma de Mexico Instituto de Fisiologia Celular

\section{Eunice Domínguez-Martin}

National Institute of Neurological Disorders and Stroke

\section{Uriel Cruz-Martínez}

Universidad Nacional Autonoma de Mexico Instituto de Fisiologia Celular

\section{Abiram E. Olivares}

Universidad Nacional Autonoma de Mexico Instituto de Fisiologia Celular

\section{Francisco Torres-Quiroz}

Universidad Nacional Autonoma de Mexico Instituto de Fisiologia Celular

\section{Laura Ongay-Larios}

Universidad Nacional Autonoma de Mexico Instituto de Fisiologia Celular

\section{Roberto Coria ( $\nabla$ rcoria@ifc.unam.mx )}

Universidad Nacional Autonoma de Mexico Instituto de Fisiologia Celular https://orcid.org/0000-00031947-5527

\section{Research Article}

Keywords: HOG pathway, UPR, Ssk1, Ssk2, glycosylation, yeast transcriptome

Posted Date: February 23rd, 2022

DOI: https://doi.org/10.21203/rs.3.rs-1347118/v1

License: (9) (1) This work is licensed under a Creative Commons Attribution 4.0 International License. Read Full License 


\section{Abstract}

In addition to the UPR pathway, yeast cells require components of the HOG pathway to respond to ER stress. In this work we found that unphosphorylated SIn1 and Ssk1 are required to mount an appropriate response to Tn. We also found that the MAPKKKs Ssk2 participates in the Tn response, but its osmoredundant protein Ssk22 does not. We also found that the Pbs2 docking sites for Ssk2 (RDS-I and KD) are partially dispensable when mutated separately, however, the prevention of Ssk2 binding to Pbs2, by the simultaneous mutation of RDS-I and KD caused strong sensitivity to Tn. In agreement with the lack of Hog1 phosphorylation during Tn treatment, a moderate resistance to $\mathrm{Tn}$ is obtained when a Pbs2 version lacking its kinase activity is expressed, however the presence of mutual Pbs2-Hog1 docking sites is essential for the Tn response. Finally, we detected that Tn induced a transcriptional activation of some components of the SLN1 branch. These results indicate that the Tn response requires a complex formed by the MAPK module and components of the SLN1 branch but not their canonical osmoregulatory activities.

\section{Introduction}

Tunicamycin ( $\mathrm{Tn})$ is one of the most used agents to induce endoplasmic reticulum (ER) stress. It is a natural antibiotic produced by Streptomyces species structurally analogous to UDP-N-acetylglucosamine. Tn inhibits the activity of Glucosamine- $N$-acetyl-phosphotransferase blocking $N$-glycosylation (Keller et al. 1979), thereby inhibits protein glycosylation and induces ER stress by accumulation of unfolded proteins. Yeast cells respond to this stress by activating the unfolded protein response (UPR) pathway that starts with the activation of the ER stress sensor Ire1, which processes the HAC1 pre-mRNA to allow its translation (Cox et al. 1993; Hernández-Elvira et al. 2018). The synthesized Hac1 transcription factor regulates transcription of genes encoding chaperons, glycosylation enzymes, and proteases, among others, to alleviate accumulation of unfolded proteins (Sidrauski 1998).

Components of other transduction pathways are required to fully cope with ER stress induced by Tn (Chen et al. 2005), including proteins of the high osmolarity glycerol pathway (HOG). The HOG pathway consists of two mechanistically different branches connected to a MAPK module (Fig. 1). The SLN1 branch and the SHO1 branch converge on the scaffold MAPKK Pbs2, which activates Hog1 under hyperosmotic stress. The first branch consists of a hybrid histidine kinase (HK) SIn1, a histidine phosphotransfer protein (HPT) Ypd1, and a response regulator (RR) Ssk1 (Salas-Delgado et al. 2017). This phosphorelay system is connected to Pbs2 through two redundant MAPKKKs, Ssk2 and Ssk22. The SHO1 branch consists of the transmembrane protein Sho1 connected to Pbs2 through a complex composed by the MAPKKK Ste11 and Ste50 (an adaptor protein for Ste11) and the PAK-like kinase Ste20 (Posas and Saito 1997).

(Figure 1) 
Previous studies have shown that yeast cells lacking Pbs2, Hog1 and Ssk1 are highly sensitive to Tn, even though they normally activate the UPR (Bicknell et al. 2010; Torres-Quiroz et al. 2010). It was also observed that the null sho1 mutant was resistant to the antibiotic. In addition to these observations, it was also determined that treatments with high concentration of Tn for long periods did not produce a significant fraction of phospho-Hog1 and did not trigger its nuclear import. In agreement with this observation, it was found that an unphosphorylated form of Hog1 (hog1TAYA in this work) was able to confer some level of resistance to Tn, however, a strain carrying Hog1 lacking its kinase activity was as sensitive as the null hog1D mutant (Torres-Quiroz et al. 2010). These data indicate that Hog1 has a cytoplasmic activity that drives cell adaptation to ER stress induced by $\mathrm{Tn}$. In line with this hypothesis, it was recently described that Hog1 may indirectly regulate extra-nuclear processes in order to induce Tn resistance, including protein glycosylation, dolichol metabolism, vesicle transport, cell wall biogenesis and detoxification systems (Hernández-Elvira et al. 2019).

In this work we made a wide exploration of the upstream Hog1 proteins that are required to confer Tn resistance. We studied the requirement of the phosphorelay system, we studied which of the MAPKKK proteins that feed into Pbs2 during the hyperosmotic shock are needed for the Tn response and we also dissected the participation of the Pbs2 scaffold domains in the Tn response

\section{Materials And Methods}

\section{Strains and mutant constructions.}

The $S$. cerevisiae deletion mutants were obtained from the EUROSCARF collection and are isogenic to the BY4742 (MATa his3 $\Delta 1$ leu2 $\Delta 0$ lys2 $\Delta 0$ ura3 $\Delta 0$ ) strain. A thermosensitive strain (TM-TS) where SIn1 is inactivated a $37^{\circ} \mathrm{C}$ was obtained from Dr. Francecs Posas. The point mutation that confers the thermosensitive phenotype (Ser694 substituted by Pro) was transferred to the BY4742 strain generating the $\sin 1$ (TS) mutant. Gene alleles containing point mutations were constructed by mutagenic PCR using oligonucleotides that introduce the desired substitutions. The mutated alleles were integrated into their chromosomal locus and/or expressed as episomic copies. Chromosomal integration was achieved by homologous recombination using the NATMX4 cassette (which provides nourseothricin resistance) as a selectable marker. Homologous recombination was achieved by including tails of 40 nt from the $5^{\prime}$ and $3^{\prime}$ UTR regions of each allele. Episomic expression was achieved by placing genes of interest under the control of the inducible galactose promoter of the pYES2 plasmid (Invitrogene) which was introduced into the corresponding null mutants. Expression of deleted genes and protein stability were confirmed by western blotting.

\section{Media and culture conditions}

YPD (1\% yeast extract, $2 \%$ glucose and $2 \%$ peptone) was used as standard growth medium. SGal (0.67\% yeast nitrogen base, $2 \%$ galactose) was used for the expression of plasmidic gene copies. Sraf was the same except that it contained $2 \%$ raffinose instead of galactose and was used to grow strains carrying plasmids. $100 \mathrm{mg} / \mathrm{ml}$ nourseothricin was added to yeast media for selection of strains carrying plasmids. 


\section{Stress Assays}

Stress sensitivity assays were done by dropping aliquots of 10-fold serial dilutions of early-logarithmic YPD-cell cultures $\left(\mathrm{OD}_{600}\right.$ adjusted to 0.5$)$ on petri dishes containing $\mathrm{Tn}$ or $\mathrm{NaCl}$. Plates were incubated at $30^{\circ} \mathrm{C}$ for $72 \mathrm{~h}$ and scanned. For liquid cultures, pre-grown cells adjusted to $\mathrm{OD}_{600} 0.05$ were inoculated by triplicate in Honeycomb 2 plates ( 100 wells). Density readings were recorded every hour during $48 \mathrm{~h}$ of incubation in a BioScreen C microplate spectrophotometer (Oy Growth Curves Ab Ltd, Helsinki, Finland). For $\operatorname{Sin} 1$ (TS) inactivation, inoculated petri dishes or Honeycomb plates were incubated at $37^{\circ} \mathrm{C}$ for $6 \mathrm{~h}$ and then transferred to $30^{\circ} \mathrm{C}$ to resume growth. For plasmidic gene copies, expression was achieved by incubation in YPGal for $4 \mathrm{~h}$ before the addition of drugs. ER stress was induced by addition of $0.7 \mathrm{mg} / \mathrm{ml}$ tunicamycin (Sigma). Hyperosmotic stress was induced with $1.0 \mathrm{M} \mathrm{NaCl}$.

\section{Protein extraction and western blot assay}

Cells were grown to midlog phase and fixed with $85 \%$ of trichloroacetic acid (TCA), collected and resuspended in loading buffer (50 $\mathrm{mM}$ Tris [pH 6.8], $100 \mathrm{mM}$ dithiothreitol (DTT), 2\% sodium dodecyl sulfate (SDS), $4 \%$ glycerol and $0.1 \%$ bromophenol blue). The suspension was then boiled at $95^{\circ} \mathrm{C}$ for 5 min and centrifuged. The soluble material was subjected to SDS polyacrylamide gel electrophoresis (SDS-PAGE) and proteins were electro-transferred to a polyvinylidene fluoride (PVDF) membrane. The membranes were probed with a polyclonal anti-Hog1 antibody, or a polyclonal anti-Pbs2 antibody. The Pbs2 KDD protein, which has lost the epitope that is recognized by the anti-Pbs2 antibody, was tagged with $6 x$ His epitope, and recognized with a monoclonal anti-6xHis antibody. Membrane bound antibodies were detected with a horseradish peroxidase-conjugated goat anti-rabbit and visualized under chemiluminescence.

\section{Gene expression}

Gene expression profiling was done by microarray analysis. Briefly, SuperAmine coated slides $(25 \times 75$ $\mathrm{mm}$, TeleChem International Inc.) were printed in duplicate with a Yeast 50-mer oligo library (representing 6529 ORFs) from MWGBiotech Oligo Sets (http:/www.mwgbiotech.com), and fixed at $80^{\circ} \mathrm{C}$ for $4 \mathrm{hrs}$. Before hybridization, slides were rehydrated, fixed with UV light and prehybridized in $5 X$ SSC, $0.1 \%$ SDS, $1 \% \mathrm{BSA}$ for one $\mathrm{h}$ at $42^{\circ} \mathrm{C}$. The first-strand cDNA (using a cDNA synthesis kit from Invitrogene) was synthetized from $10 \mu \mathrm{g}$ of total RNA of cells treated or not treated with $2 \mu \mathrm{g} / \mathrm{ml} \mathrm{Tn}$ for $60 \mathrm{~min}$. The cDNA was labeled with dUTP-Alexa555 or dUTP-Alexa647. Equal quantities of labeled cDNA from two biological replicates were hybridized using UniHyb solution (TeleChem International Inc) with the collection of oligo-arrays for $14 \mathrm{~h}$ at $42^{\circ} \mathrm{C}$. Microarray data analysis was performed with free software genArise, developed by the Computing staff of the Cellular Physiology Institute, UNAM (http://www.ifc.unam.mx/genarise/). Metabolic pathways were analyzed with KEGG pathway database (https://www.kegg.jp/kegg/pathway.html). Expression heat maps were constructed with the $\log _{2}$ of the net intensity values (supp table 1) using the Graph pad Prism 9, V 9.2.0 (283) program.

\section{Other}


Standard techniques for yeast genetics and recombinant DNA were performed in this work. All PCR products were sequenced before homologous integration and expression. Integrated mutant alleles were confirmed by PCR.

\section{Results}

Table 1 summarizes our findings regarding the participation of the HOG pathway in the Tn response. We assigned values of Tn resistance ranging from 10 for the wild type strain to 0 for the highly sensitive ire1D mutant. For comparison we included in our analysis the resistance values to $\mathrm{NaCl}$ which induces hyperosmotic stress. We divided our strains in three groups, those belonging to the SLN1 branch, those of the SHO1 branch and the Pbs2-Hog1 module.

(Table 1)

To determine whether the SIn1 histidine kinase participates in the Tn response we expressed a thermosensitive protein $\left(\sin 1(T S)\right.$, which is inactivated at $\left.37^{\circ} \mathrm{C}\right)$ in a strain carrying the hog 1 TAYA protein. This protein lacks the Thr-174 and Tyr-176 residues subjected to phosphorylation by Pbs2. It is important to recall that a strain expressing the hog 1TAYA allele is highly sensitive to $\mathrm{NaCl}$, but most importantly, partially resistant to $\mathrm{Tn}$ (Torres-Quiroz et al. 2010). In this strain, the inactivation of $\operatorname{Sin} 1$ does not induce the lethality otherwise induced by the constitutive activation of Hog1 wild type protein (Posas et al. 1996). When we analyzed growth of the $\sin 1(T S) /$ hogTAYA strain and compared it with the growth of the hogTAYA strain we found that inactivation of $\operatorname{Sin} 1$ at $37^{\circ} \mathrm{C}$ caused a significant decrease of the $\mathrm{Tn}$ resistance (Table1). To analyze the contribution of the phosphorelay activity of $\operatorname{Sin} 1$ we followed the same strategy. We expressed, in the $\sin 1(T S) / \operatorname{hog} 1 T A Y A$ strain, the $\sin 1 H Q$ allele (in which the phosphorylatable His-576 residue has been substituted by GIn); this protein is deficient in autophosphorylation and cannot transfer phosphate groups to its receiver domain (Fig. 1) (Posas et al. 1996). We found that the sensitivity of the $\sin 1(T S)$ strain is complemented by the $\sin 1 H Q$ allele reaching a value near the wild type strain (Table 1). This result indicates that the unphosphorylated form of $\operatorname{Sln} 1$ is participating in the Tn response.

We also analyzed the contribution of the response regulator Ssk1. First, we confirmed the previous observation that a null ssk1D mutant shows sensitivity to Tn (Table1) (Torres-Quiroz et al. 2010). We then explored the contribution of the Asp-554 residue to the Tn response. The substitution of Asp-554 by an Ala residue (ssk1DA) prevents the Ypd1-dependent phosphorylation of Ssk1 (Fig. 1). We observed that the expression of the ssk1DA protein in the ssk1D strain, reverted the Tn sensitivity to the value near to that of the wild type strain. Finally, we determined the effect that elimination of the MAPKKKs Ssk2 and Ssk22 have in the Tn response. Elimination of Ssk2 significantly affected the growth in Tn medium, however elimination of Ssk22 had a much weaker effect. Elimination of both Ssk2 and Ssk22 moderately increased the sensitivity shown by the ssk2D mutant. This observation strongly suggests that Ssk2 plays a prominent role in the Tn response and disregards the participation of Ssk22. 
In this work we also extended the studies regarding the participation of components of the SHO1 branch (Table 1). We confirmed that elimination of Sho1 did not affect growth in Tn and accordingly we found that elimination of the MAPKKK Ste11 had no effect either. Elimination of the adaptor protein Ste50 or the PAK-like kinase Ste20 barely induced a slight reduction in Tn resistance. The different effect that lack of these proteins had in the Tn response compared to the lack of Ste11 could be due to their participation in other transduction pathways.

Previous studies on the participation of components of the MAP kinase module in the response to inducers of ER stress, indicated that disruption of Hog1 and Psb2 caused high sensitivity to Tn (Bicknell et al. 2010; Torres-Quiroz et al. 2010). We confirmed those observations and found that the sensitivity of the pbs2D mutant was stronger than that of the hog1D mutant (Table 1). In addition, our quantitative studies showed that the expression of the unphosphorylated form of Hog1 (hog1TAYA) confers about three-fold resistance to Tn compared to the hog1 null mutant.

Then we analyzed the role of the scaffold domains of $\mathrm{Pbs} 2$, that have previously been described (Tatebayashi et al. 2003). To this end we constructed various Pbs2 mutants that compromise its association with Ssk2, Sho1 and Hog1. We also constructed a point mutation where the kinase activity of Pbs2 was eliminated (Fig. 2A). In these studies, we included a Hog1 version with the Pbs2BD domain deleted (Fig. 2B), which comprises the adjacent CD and PBD-2 sites required for Pbs2 binding (Murakami et al. 2008). All constructions were either integrated into their chromosomal loci, which places the mutated genes under the control of their endogenous promoter or cloned into the pYES2 vector and expressed under the control of the GAL 1 promoter. In our experiments, we did not detect growth differences between strains carrying the integrated or the episomic copies.

Our results show that elimination of the Ssk2/22 binding domain (RSD-I, 5-56 aa) slightly reduced Tn resistance, while substitution of the Val54 residue for Gly, located within this domain (Fig. 2A), had no effect in the Tn resistance, which is comparable to the wild type strain (Table 1). This V54G mutation prevents binding of Ssk2/22 to the isolated RSD-I domain (Tatebayashi et al. 2003). Mutants lacking the Sho1 binding domain (RSD-II, 55-107 aa) showed a very light reduction of Tn resistance (Table 1).

(Figure 2)

We then analyzed the role of the Pbs2 kinase domain (KD), which has also a docking motif for Ssk2 (Tatebayashi et al. 2003); we found that a Pbs2 protein with the KD domain deleted (KD, 353-669 aa) still conferred significant resistance to Tn. Similarly, a strain carrying a Pbs2 protein devoid of kinase activity, pbs2KM (Lys-389 substituted by Met) (Fig. 2A), displayed mid resistance to Tn (Table1). It has been reported that simultaneous elimination of the KD domain and the substitution of Val-54 by Gly completely abrogates the interaction of Pbs2 with Ssk2 in the hyperosmotic system (Tatebayashi et al. 2003). In our assays we found that the double mutation KDD/V54G caused a strong Tn sensitivity; in this mutant the Tn resistance dropped to the level shown by the pbs2D null mutant (Table1). 
To complement the Pbs2 domain dissection we analyzed the effect of the elimination of the HBD-1 (136$245 \mathrm{aa}$ ) domain of Pbs2 (Fig. 2A), which is a binding domain for Hog1 (Murakami et al. 2008). In our experiments, deletion of this domain caused a strong reduction on the Tn resistance (Table 1), and this effect was similar to the effect that elimination of the Hog1 Pbs2BD domain had in Tn resistance. The Hog1 Pbs2BD deletion includes the two binding domains described as Pbs2 docking motifs (Murakami et al. 2008). These results demonstrate that binding of Pbs2 and Hog1 is an essential requirement for the response to Tn.

Finally, to gain insight into the transcriptional effect that Tn treatment elicit in yeast, total RNA was extracted from cells exposed to Tn as indicated in the methods section and hybridized with an oligo-array representing 6529 ORFs. The result of the microarrays analysis is depicted in supplementary table S1. The $\log _{2}$ value of the differential expression between cells treated with $\mathrm{Tn}$ and not treated was calculated and used to generate heat maps of expressed (or repressed) genes belonging to three pathways annotated in KEGG: the MAPK (HOG) pathway, the protein processing in the ER pathway, and various types of N-Glycan biosynthesis pathway (Fig. 3). As expected, we detected that several genes of the UPR and N-Glycosylation pathways were induced under Tn treatment (Fig. 3B, C). Interestingly, Tn increased transcripts abundance of genes whose products participate in the SLN1 branch of the HOG pathway, namely SLN1, SSK2, SSK22, and YPD1 (Fig. 3A). In this assay expression of SSK1 was unchanged. It is also interesting to note that the expression of $P B S 2$ was slightly increased while HOG1 was repressed. In this study we did not detect significant expression changes in components of the SHO1 branch (table S1).

(Figure 3)

\section{Discussion}

The MAPK Hog1 controls stress responses that do not always overlap with the response to hyperosmotic stress. The sensing mechanism often employs one branch at a time, more commonly the SLN1 branch, which appears to have the plasticity to adapt to different stimuli. Here we have determined the requirement of the SLN1 branch for the cell response to ER stress induced by Tn. In this work we determined that $\operatorname{SIn} 1$ and Ssk1 are required to achieve an optimal response to Tn, however the residues involved in the phosphate transfer are dispensable. This would indicate that the pathway of Tn response is independent of the phosphotransfer system and similar to the hyperosmotic pathway, where the unphosphorylated Ssk1 would be the active form (Posas et al. 1996; Horie et al. 2008). Our results also indicate that the connection of the two-component system with the Pbs2-Hog1 tier is mediated by the MAPKKK Ssk2, and that Ssk22 plays a less prominent role. This suggests that Ssk2 and Ssk22 are not redundant for the Tn response, as they are for the hyperosmotic pathway (Maeda et al. 1995).

In our studies we consistently found that the pbs2D mutant was more sensitive to Tn than the hog1D. This phenotype is consistent with previous observations where a pbs2D mutant ranked within a group of highly sensitive mutant strains to both Tn and b-mercaptoethanol (Chen et al. 2005). We extended these 
observations with the study of the structural associations of Pbs2 with upstream and downstream elements. The differential scaffolding participation of Pbs2 in hyperosmotic stress has been dissected previously. In those studies, the kinase domain (KD) and the binding domains for Ssk2/22 (RDSI), Sho1(RDSII) and Hog1(HBD-1) were described (Tatebayashi et al. 2003; Murakami et al. 2008).

Our results point to a model where the scaffold activity of Pbs2 is essential for the Tn response, but not its kinase activity. At least two structural elements play prominent roles: One is the physical binding of Ssk2 with the docking sites present in the RDS-I and KD domains of Pbs2. This Ssk2-Pbs2 binding model (Tatebayashi et al. 2003), suggests that in the inactive state, the kinase domain of Ssk2 binds to the RDSl; then under hyperosmotic shock, Ssk1 associates with Ssk2. This makes the Ssk2 kinase domain accessible for the binding of the Pbs2 KD domain. This mechanism of signal transmission could be conserved for the Tn response, as evidenced by the strong Tn sensitivity displayed by the double KDD/V54G mutant. The second is the molecular association of Pbs2 and Hog1. Our results indicate that strong sensitivity to $\mathrm{Tn}$ is obtained when the Hog1 binding domain in Pbs2 or the Pbs2 binding domain in Hog1 are eliminated. This indicates that Pbs2 and Hog1 act as a complex during the Tn exposure.

With our studies, we conclude that the cellular response to Tn requires a complex formed by elements of the SLN1 branch associated with the MAPK module Pbs2-Hog1. In this system, the SHO1 branch elements are dispensable. Although the structural components may be conserved between the suggested Tn response complex and the hyperosmotic response complex, the canonical activities needed for osmoregulation are not necessarily required for $\mathrm{Tn}$ adaptation. The experimental evidence suggests that proteins of the HOG pathway form a pre-activation complex in the isosmotic state. This opens the question whether this is in fact, the active Tn response complex and what is the nature of the stimulus imposed by $\mathrm{Tn}$ that will activate this complex. The actual model for regulation of $\operatorname{SIn} 1$ kinase activity suggests that changes in turgor caused by hyperosmotic shock will reduce the SIn 1 kinase activity (Tao et al. 2002; Reiser et al. 2003). According with this model, we can hypothesize that the drop in the concentration of glycosylated proteins caused by $\mathrm{Tn}$, will exert pressure against the cell wall affecting mechanically the activity of $\operatorname{SIn} 1$. A second hypothesis that can be raised is that the $T n$ response is independent of turgor. In this scenario, the Tn treatment may affect the concentration of specific cell wall proteins that can regulate the SIn 1 activity. One example is the GPI-anchored cell wall mannoprotein Ccw12 that has been described as a modulator of SIn1 kinase activity (Narang et al. 2008). In agreement with this second hypothesis it has been shown that Hog1 indirectly regulates proteins that participate in cell wall biogenesis (Hernández-Elvira et al. 2019), such as the Glutamine-fructose-6-phosphate amidotransferase Gfa1. This protein participates in the first step of chitin biosynthesis.

In this study, we detected that Tn triggers expression of genes involved in protein folding, glycosylation and degradation which are processes typically activated upon ER stress (Cox et al. 1993). Interestingly the SLN1 branch of the HOG pathway is also upregulated by increased transcription of SLN1, YPD1 and SSK2, but not SSK1, thus yeast adapt to the Tn stress by transcriptional reprogramming of some HOG pathway components. PBS2 and HOG1 were not subjected to upregulation by $\mathrm{Tn}$, suggesting that basal concentration of these proteins is sufficient to mount the appropriate response. 
Tn is considered a highly specific inhibitor of protein glycosylation, an ER resident process that influences many aspects of cellular metabolism, while the HOG complex is tethered to the plasma membrane. Physical contact between cortical ER and plasma membranes (PM) occurs through membrane contact sites (MCS) that are stablished through ER integral membrane proteins (Zaman et al. 2020), thus, it is possible that a tight coordination exits between ER and PM at the MCS to regulate responses to the membrane stress imposed by Tn, in one hand the UPR mediated by the Ire 1 sensor and in the other the two-component SIn 1 histidine kinase-MAPK module.

In this work, we have advanced significantly in the understanding of the structural and functional elements that build the yeast Tn response pathway, although some questions remain open and pending to be answered.

\section{Declarations}

\section{Acknowledgments}

We thank Francesc Posas for the SIn1(TS) strain. GS-D is a PhD student of the Biochemical Sciences Program, UNAM. We acknowledge the technical support provided by Guadalupe Codiz and Minerva Mora from the Molecular Biology Unit; Gerardo Coello, Ana Ma. Escalante, Juan Barbosa and Ivette Rosas from the Computing Unit; Aurey Galván and Manuel Ortínez from the Maintenance Workshop. The microarray analysis was performed in the Microarray Unit (IFC, UNAM). We thank Lorena Chávez González, Simón Guzmán León, and Jorge Ramírez for technical assistance in the microarray determinations. We also acknowledge the technical assistance of Ma. Teresa Lara Ortíz.

\section{Author Contributions}

MH-E, LK, GS-D, ED-M, UC-M and AEO performed experiments. FT-Q, LO-L, and RC conceived the project. MH-E, LK, GS-D, ED-M, and RC designed experiments and analyzed the data. ED-M designed and performed the microarray analysis. FT-Q and RC supervised the project. MH-E and RC wrote the first draft of the manuscript. LO-L and RC wrote the final version of the manuscript with comments from all authors.

\section{Funding}

This work was supported in part by grands DGAPA-PAPIIT No. IN210519 and CONACyT No. CF-58550.

Conflicts of Interest: The authors declare no conflicts of interest.

Consent for publication: All authors agreed to the published version of the manuscript

\section{References}

1. Bicknell AA, Tourtellotte $\mathrm{J}$ and Niwa M (2010) Late Phase of the Endoplasmic Reticulum Stress Response Pathway Is Regulated by Hog1 MAP Kinase. J Biol Chem 285, 17545-17555. 
https://doi.org/10.1074/jbc.m109.084681

2. Cox JS, Shamu CE and Walter $P$ (1993) Transcriptional induction of genes encoding endoplasmic reticulum resident proteins requires a transmembrane protein kinase. Cell 73, 1197-1206. https://doi.org/10.1016/0092-8674(93)90648-a

3. Chen Y, Feldman DE, Deng C, Brown JA, De Giacomo AF, Gaw AF, Shi G, Le QT, Brown JM and Koong AC (2005) Identification of Mitogen-Activated Protein Kinase signaling pathways that confer resistance to endoplasmic reticulum stress in Saccharomyces cerevisiae. Mol Cancer Res 3, 669-677. https://doi.org/10.1158/1541-7786.mcr-05-0181

4. Hernández-Elvira M, Torres-Quiroz F, Escamilla-Ayala A, Domínguez-Martin E, Escalante R, Kawasaki L, Ongay-Larios L and Coria R (2018) The unfolded protein response pathway in the yeast Kluyveromyces lactis. A comparative view among yeast species. Cells 7, 106. https://doi.org/10.3390/cells7080106

5. Hernández-Elvira M, Martínez-Gómez R, Domínguez-Martin E, Méndez A, Kawasaki L, Ongay-Larios L and Coria R (2019) Tunicamycin sensitivity-suppression by high gene dosage reveals new functions of the yeast Hog1 MAP kinase. Cells 8, 710. https://doi.org/10.3390/cells8070710

6. Horie T, Tatebayashi K, Yamada R and Saito H (2008) Phosphorylated Ssk1 prevents unphosphorylated Ssk1 from activating the Ssk2 mitogen-activated protein kinase kinase kinase in the yeast high osmolarity glycerol osmoregulatory pathway. Mol Cell Biol 28, 5172-5183. https://doi.org/10.1128/mcb.00589-08

7. Keller RK, Boon DY and Crum FC (1979) N-Acetylglucosamine-1-phosphate transferase from hen oviduct: solubilization, characterization, and inhibition by tunicamycin. Biochemistry 18, 3946-3952. https://doi.org/10.1021/bi00585a016

8. Maeda T, Takekawa M and Saito H (1995) Activation of yeast PBS2 MAPKK by MAPKKKs or by binding of an SH3-containing osmosensor. Science 269, 554-558. https://doi.org/10.1126/science.7624781

9. Murakami Y, Tatebayashi K and Saito H (2008) Two adjacent docking sites in the yeast Hog1 mitogen-activated protein (MAP) kinase differentially interact with the Pbs2 MAP kinase kinase and the Ptp2 protein tyrosine phosphatase. Mol Cell Biol 28, 2481-2494. https://doi.org/10.1128/mcb.01817-07

10. Narang SS, Malone CL, Deschenes RJ and Fassler JS (2008) Modulation of yeast SIn1 kinase activity by the Ccw12 cell wall protein. J Biol Chem 283, 1962-1973. https://doi.org/10.1074/jbc.m706877200

11. Posas F, Wurgler-Murphy SM, Maeda T, Witten EA, Thai TC and Saito H (1996) Yeast HOG1 MAP kinase cascade is regulated by a multistep phosphorelay mechanism in the SLN1-YPD1-SSK1 "twocomponent" osmosensor. Cell 86, 865-875. https://doi.org/10.1016/s0092-8674(00)80162-2

12. Posas $F$ and Saito H (1997) Osmotic activation of the HOG MAPK pathway via Ste11p MAKKK: scaffold role of Pbs2p MAPKK. Science 276, 1702-1705. https://doi.org/10.1126/science.276.5319.1702 
13. Reiser V, Raitt DC and Saito H (2003) Yeast osmosensor SIn1 and plant cytokinin receptor Cre1 respond to changes in turgor pressure. J Cell Biol 161, 1035-1040. https://doi.org/10.1083/jcb.200301099

14. Salas-Delgado G, Ongay-Larios L, Kawasaki-Watanabe L, López-Villaseñor I and Coria R (2017) The yeasts phosphorelay systems: a comparative view. World J Microbiol Biotechnol 33, 111. https://doi.org/10.1007/s11274-017-2272-z

15. Sidrauski C (1998) The unfolded protein response: An intracellular signaling pathway with many surprising features. Trends Cell Biol 8, 245-249. https://doi.org/10.1016/s0962-8924(98)01267-7

16. Tao W, Malone CL, Ault AD, Deschenes RJ and Fassler JS (2002) A cytoplasmic coiled-coil domain is required for the histidine kinase activity of the yeast osmosensor, SIn1. Mol Microbiol 43, 459- 473. https://doi.org/10.1046/j.1365-2958.2002.02757.x

17. Tatebayashi K, Takekawa M and Saito H (2003) A docking site determining specificity of Pbs2 MAPKK for Ssk2/Ssk22 MAPKKKs in the yeast HOG pathway. EMBO J 22, 3624-3634. https://doi.org/10.1093/emboj/cdg353

18. Torres-Quiroz F, García-Marqués S, Coria R, Randez-Gil F and Prieto JA (2010) The activity of yeast Hog1 MAPK is required during endoplasmic reticulum stress induced by tunicamycin exposure. $J$ Biol Chem 285, 20088-20096. https://doi.org/10.1074/jbc.m109.063578

19. Zaman MF, Nenadic A, Radojičić A, Rosado A and Beh CT (2020) Sticking with it: ER-PM membrane contact sites as a coordinating nexus for regulating lipids and proteins at the cell cortex. Front Cell Dev Biol 8, 675. https://doi.org/10.3389/fcell.2020.00675

\section{Tables}

Table 1. List of analyzed mutants showing $\mathrm{Tn}$ and $\mathrm{NaCl}$ resistance values. 
gene

mutant

observation

Tn

resistance

$\mathrm{NaCl}$

resistance

WT

WT

Parental strain

10

10

\section{SLN1 branch}

\begin{tabular}{|c|c|c|c|}
\hline \multirow[t]{2}{*}{ SLN1 } & $\sin 1(T S)$ & $\begin{array}{l}\text { Sln } 1 \text { inactivation at } 37^{\circ} \mathrm{C} \text {. Expresion in } \\
\text { hog } 1 \text { TAYA backgroung. }\end{array}$ & 3 \\
\hline & $\sin 1 H Q$ & $\begin{array}{l}\sin 1 \mathrm{HQ} \text { expresion in } \\
\sin 1(\mathrm{TS}) / \mathrm{hog} 1 \mathrm{TAYA} \text { backgroung at } \\
37^{\circ} \mathrm{C}\end{array}$ & 7 \\
\hline
\end{tabular}

SSK 1

ssk1D

full ORF deleted

3

10

ssk1DA

Substitution of Asp554 by Alanine

8

10

SSK2/SSK22

ssk2D

full ORF deleted

5

10

ssk22D

full ORF deleted

8

10

ssk2D/ssk22D

full ORFs deleted

4

10

\section{SHO1 branch}

SHO1
STE11
STE5O
STE2O
MAPKK-MAPK

\begin{tabular}{|c|c|c|c|c|}
\hline \multirow[t]{8}{*}{ PBS2 } & $p b s 2 D$ & full ORF deleted & 1 & 0 \\
\hline & $R S D-I D$ & 5-56 aa deleted & 7 & 0 \\
\hline & $R S D-I I D$ & 55-107 aa deleted & 9 & 2 \\
\hline & $H B D-1 D$ & 136-245 aa deleted & 3 & 2 \\
\hline & $K D D$ & $353-668$ aa deleted & 6 & 0 \\
\hline & $p b s 2 K M$ & Substitution of Lys389 by Met & 5 & 0 \\
\hline & V54G & Substitution of Val54 by Gly & 10 & 10 \\
\hline & $K D D / \mathrm{V} 54 \mathrm{G}$ & $\begin{array}{l}353-668 \text { aa deleted }+ \text { Substitution of } \\
\text { Val } 54 \text { by Gly }\end{array}$ & 1 & 0 \\
\hline \multirow[t]{2}{*}{ HOG1 } & $\operatorname{hog} 1 D$ & full ORF deleted & 2 & 0 \\
\hline & hogTAYA & $\begin{array}{l}\text { Substitution of Thr174 and Tyr176 by } \\
\text { Ala }\end{array}$ & 6 & 0 \\
\hline
\end{tabular}


UPR pathway

IRE1

ire1D

full ORF deleted

0 10

Note: Single deletion mutants were obtained from the EUROSCARF collection and are isogenic to the BY4742 strain. Double deletion and nucleotide substitution mutants were constructed as indicated in Methods section. Relative resistance values were obtained from quantitative growing assays and referred to the wild type strain which shows the highest growing rates in $\mathrm{Tn}$ and $\mathrm{NaCl}$. ER stress was induced by addition of $0.7 \mathrm{mg} / \mathrm{ml}$ tunicamycin. Hyperosmotic stress was induced with $1.0 \mathrm{M} \mathrm{NaCl}$.

Figures

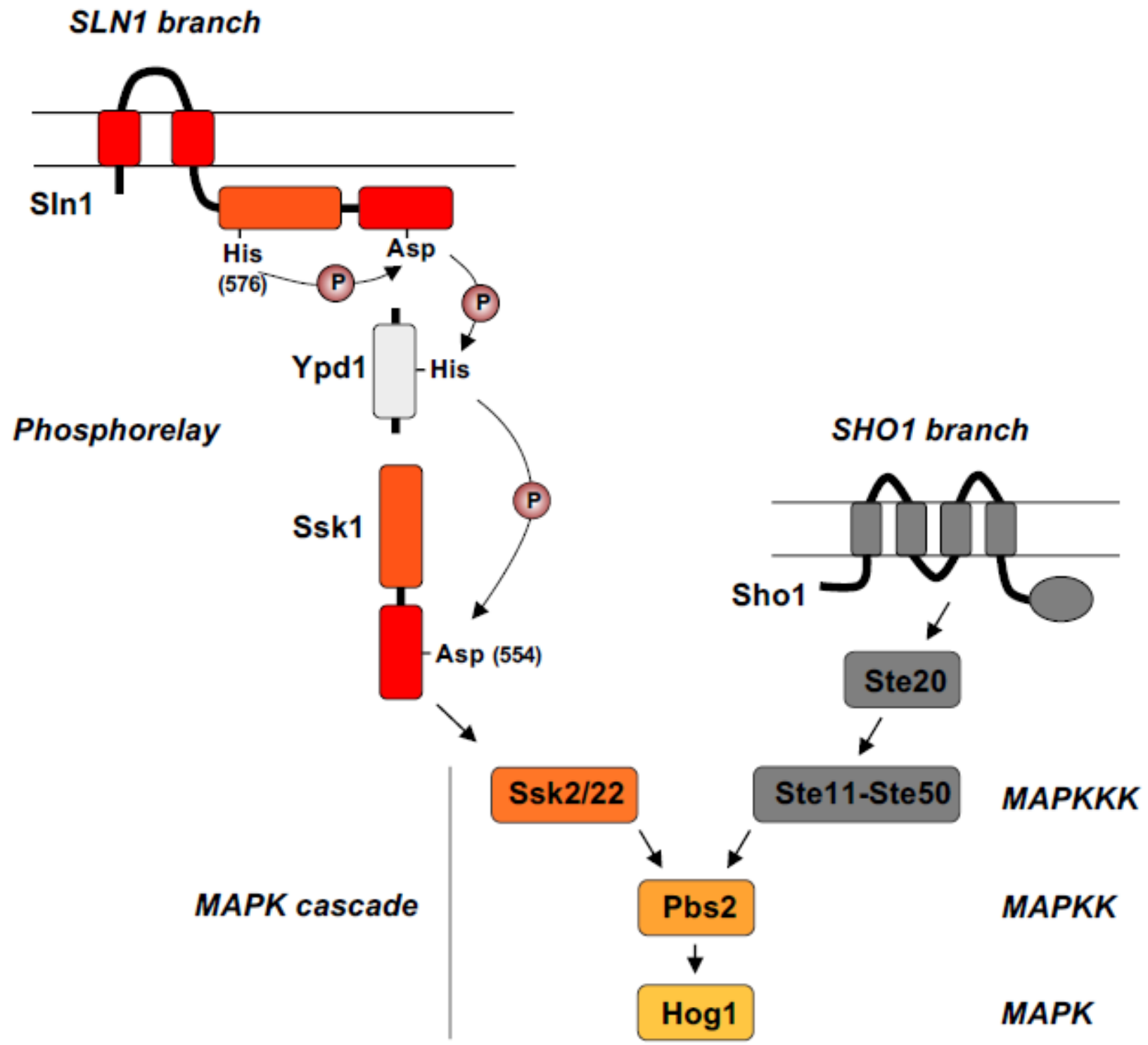

Figure 1 
Schematic representation of the HOG pathway. For simplification only relevant elements for this study are shown. Proteins involved in the Tn response are shown in orange tones. Ypd1 was not analyzed in this study. Amino acids involved in the phosphotransfer reaction are indicated. His-576 was substituted by GIn in SIn1 to yield s/n1HQ. Asp-554 was substituted by Ala in Ssk1 to yield ssk1DA.

\section{A}
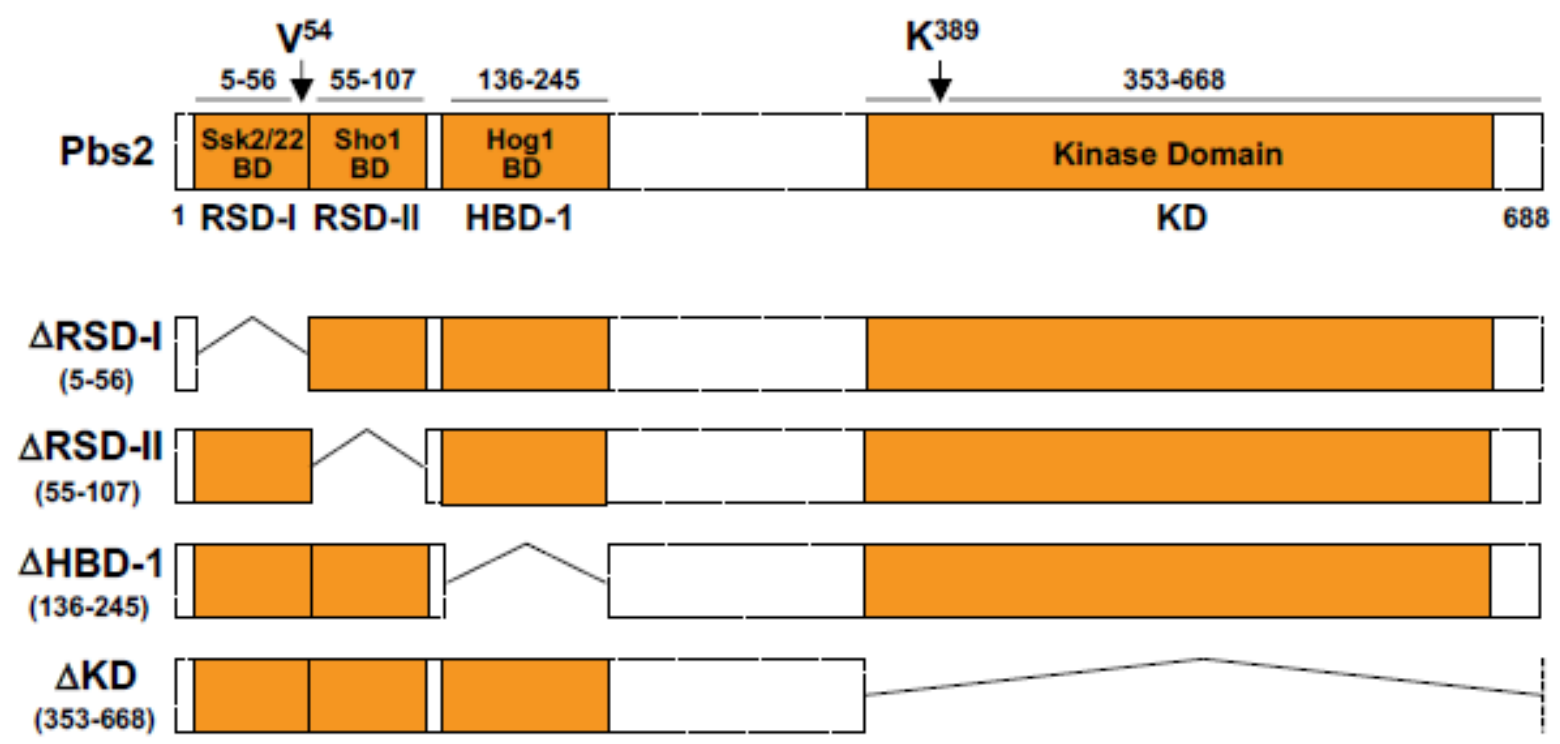

B

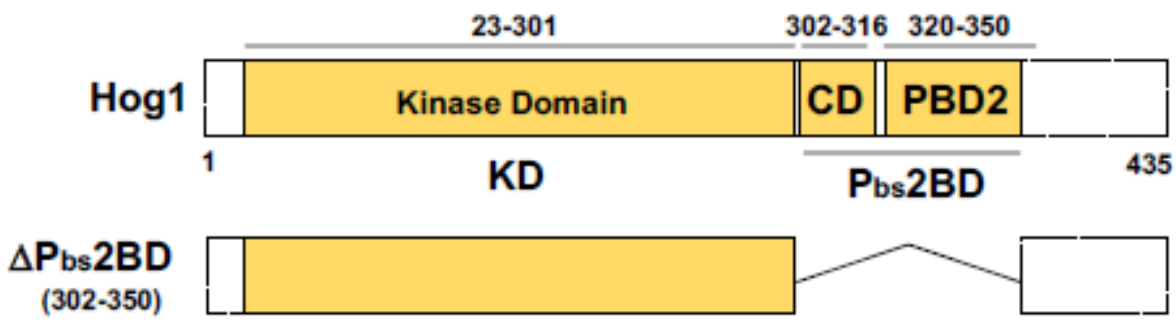

\section{Figure 2}

Schematic representation of Pbs2 and Hog1 and their deletion derivatives constructed for this study. Domains were named according to references Tatebayashi et al. (2003) and Murakami et al. (2008). A) Full-length Pbs2 showing the domains and amino acids analyzed. Val-54 (V) lays withing RSD-I domain and was substituted by Gly to construct V54G. Lys-389 (K) is the catalytic residue and was substituted by Met $(\mathrm{M})$ to construct mutant pbs2KM. B) Full-length Hog1 showing relevant domains. The CD (Common Domain) and the Pbs2 Binding Domain were eliminated to construct DPbs2BD mutant. 

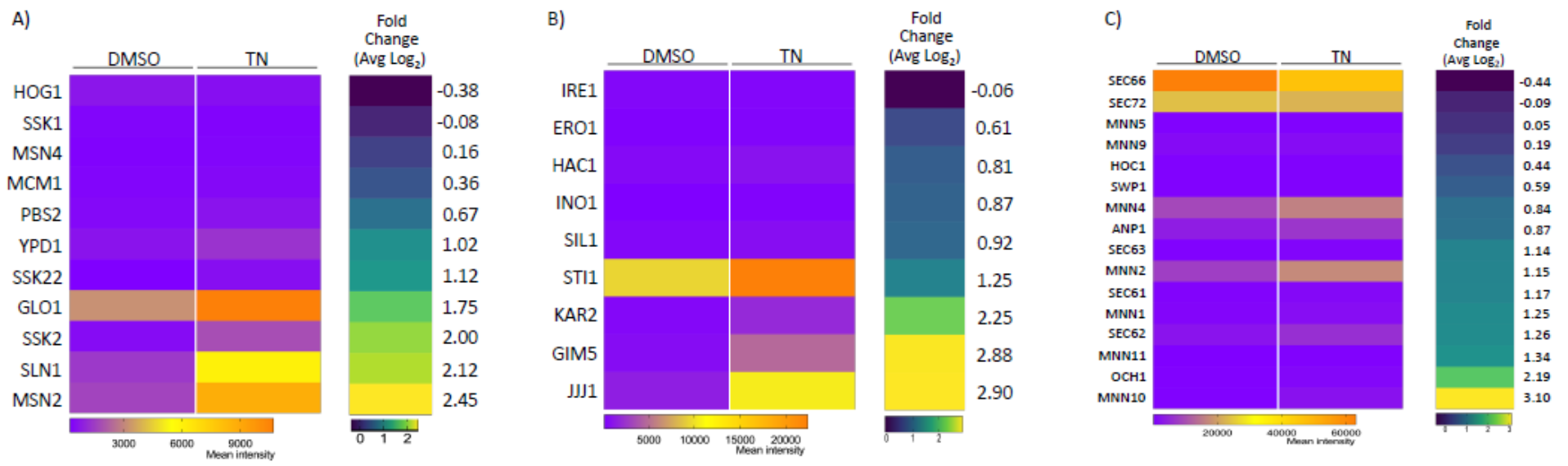

\section{Figure 3}

Gene expression fold change of cells treated (Tn) and not treated (DMSO) represented as heat maps. The fold change is expressed as the $\log _{2}$ value of the average net intensity of two biological replicates. Genes were categorized into the respective metabolic pathway according to the KEGG database; (A) MAPK (HOG) signaling pathway (Sce04011); (B) Protein processing in the ER (Sce04141); (C) Various types of N-Glycan biosynthesis (Sce00513).

\section{Supplementary Files}

This is a list of supplementary files associated with this preprint. Click to download.

- TableS1ScBY4741MicroarraysDMSOTN.xls 